\title{
The Availability of Systems with Bathtub Hazard Rate Function
}

\author{
M.Y. Ashkar \\ Department of Mathematics, University of Bahrain, Kingdom of Bahrain. \\ myashkar@hotmail.com
}

\begin{abstract}
:
In our normal life we can see that the most realistic systems possess useful time governed by hazard rate of bathtub shaped. The hazard rate function, however, plays a vital role in the computation of the availability function. The repair time, however, could be modeled as any statistical distribution. In this paper I will investigate the nature of availability function and points of availability of systems with bathtub hazard function and exponential distribution repair time using Markovian method.
\end{abstract}

\section{Introduction}

When a system of multi-units or a single unit is put into service, the availability or point-availability of a system or item is very important for its useful life time. And before it breaks down and is sent to be repaired. However, it is important to know about how many times the device (system) has been replaced or is repaired after failure before reaching the asymptotic state. The device may be replaced many times or it could have never been replaced at all. The device's reliability measurement is still important and needed. So, the relation between reliability, maintainability and/or availability is vital and momentous, Kapur [13] and Leemis [21].

The life-time cycle of a repairable system, can be described by a sequence of up and down states, i.e. working and under repair. So, the system operates until it fails, then it is repaired (or replaced) and is returned to its original operating state. It may fail again after some random span time of operation. If so, get it repaired again. This process of failure and repair will be repeated for a sufficient enough amount of time and is called a renewal process. The renewal process is a sequence of independent random variables, Cox [7]. In this case, the random variables are the times to failure and the times to repair. At each time when a device fails and is repaired, a renewal is said to have occurred. This type of renewal process is known as an Alternating Renewal Process because the state of the device alternates between a functioning (up-time) and under repair (down-time), Cox [7].

One of the main assumptions in the renewal theory is that the failure items are replaced or repaired with new ones so they are "as good as new" hence the name 'renewal' is obtained, Kijima [16], [17], Elsayed [11] and Leemis [21].

The definition of availability is rather flexible and is widely based on the types of down-times, or under repair, one chooses to consider in the analysis. There are different number of classifications of availability, such as, Instantaneous availability, average up-time availability and steady state (Asymptotic) Availability, Kececioglu [14], [15].

The immediate availability, or point availability is defined as the probability that a system will be in operation at any random time, t. Unlike reliability, the instantaneous availability measure incorporates maintainability information. At any given time, $t$, the system will be operational if the following conditions are met, Elsayed [11], Kececioglu [15].

The item functioned properly from 0 to $t$ with probability $R(t)=1-F(t)$ or it functioned properly since the last repair at time $\mathrm{u}, 0<\mathrm{u}<\mathrm{t}$, with probability 


$$
\int_{0}^{t} R(t-u) m(u) d u
$$

where $m(u)$ is the renewal density function of the system. Hence, then the point availability is given by

$$
P A(t)=R(t)+\int_{0}^{t} R(t-u) m(u) d u
$$

, Cox [7]. The latest PA(t) is a Volterra's integral equation of the second kind, Fox [12]. It can be solved numerically and it can be applied for systems without singularities. So, we have to find out a method to cope with a very wide class of distributions regardless their scale and position parameters. The method could be applied to Gamma, Weibull, Lognormal and most of two parameters distributions. Also, it can be used to find the point availability of systems of Bathtub shaped hazard rate. But here, the method will be applied strictly to systems of Bathtub shaped hazard rate.

\section{The Bathtub Hazard Rate Function:}

The bathtub curve that describes a particular form of the hazard rate function is widely used in reliability. This curve comprises three major parts, decreasing failure rate, constant failure rate and finally an increasing failure rate. The name is derived from the cross-sectional shape of a bathtub. This curve is represented in figure 1 .

In detail, the bathtub curve is generated by mapping the rate of early "infant mortality" failures when first introduced and put into service, the rate of random failures with constant failure rate during its "useful life" under warranty period, and finally the rate of "wear out" failures as the product exceeds its design lifetime.

The hazard rate function of probabilistic failure can be found directly from the reliability function, which is obtained from the probability density function of the up-time.

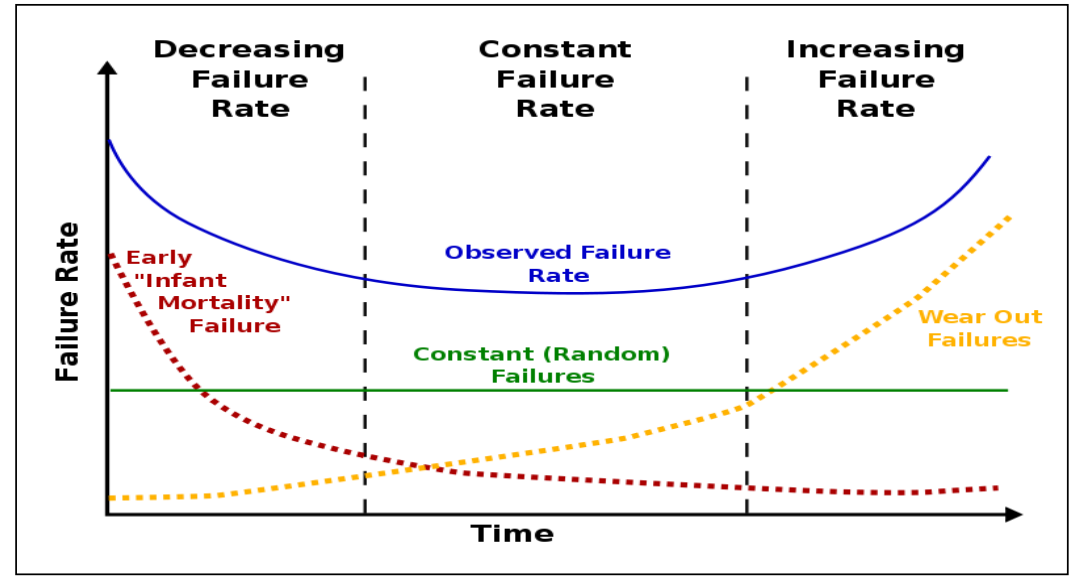

Figure1 The General Bathtub Curve.

(en.wikipedia.org /Bathtub curve)

We can, in reverse, use the hazard rate as the starting point. In this case we have to consider the hazard rate $h(t)$ of a type similar to that shown in figure 1. This is known as "A Bathtub curve". During the "Burnin" period the hazard rate $h(t)$ decreases, since a proportion of manufactured components contain weaknesses which cause early failure. After a while, the early failures are weeded out and $h(t)$ commences a nearly constant failure rate period.

This constant failure period is known as the "useful life". It allows a chance of failure by sudden stress, perhaps caused by unusually severe and unpredictable operating conditions. To eliminate these 
unpleasant circumstances, it would require a device that is overdesigned for the vast majority of situations. Eventually, the item starts its third period and the hazard rate $h(t)$ increases as the item "wears out", resulting from equipment deterioration of cumulated shocks, fatigue, and alike. By assuming that in these three regions the failure rate is linearly modeled, we can specify the hazard rate for each region.

Having in mind that the three regions are respectively, $0 \leq t<t_{1}, t_{1} \leq t<t_{2}$ and $t_{2} \leq t<\infty$, see Shooman [24].

In the first region the hazard rate is given as

$$
h(t)=a_{1}-b_{1} t
$$

In the second and third regions the hazard rate is given respectively as

$$
\begin{array}{ll}
h(t)=a_{2}-b_{2}\left(t-t_{1}\right) & t_{1} \leq t \leq t_{2} \\
h(t)=a_{3}-b_{3}\left(t-t_{2}\right) & t_{2} \leq t \leq+\infty
\end{array}
$$

Then the slope can be calculated

$$
b_{1}=\frac{a_{1}-a_{2}}{t_{1}-0} \quad b_{2}=\frac{a_{2}-a_{3}}{t_{2}-t_{1}} \quad b_{1}=\frac{a_{4}-a_{3}}{t_{3}-t_{2}}
$$

Note that if $a_{2} \cong a_{3}$ then $\mathrm{b} 2=0$.

3 Reliability function:

We define the reliability functioning as

$$
R(t)=e^{-\int_{0}^{t 0} h(v) d v}
$$

So, the reliability function related to Bathtub hazard rate will be given as

$$
R(t)= \begin{cases}e^{-\left(a_{1} t-\frac{1}{2} t^{2}\right)} & 0 \leq t<t_{1} \\ e^{-\left(a_{1} t_{1}-\frac{1}{2} t^{2}\right)} e^{-a_{2}\left(t-t_{1}\right)} & t_{1} \leq t<t_{2} \\ e^{-\left(a_{1} t_{1}-\frac{1}{2} t^{2}\right)} e^{-a_{2}\left(t_{2}-t_{1}\right)} e^{-\left[a_{3}\left(t-t_{2}\right)+b_{3}\left(t-t_{2}\right)^{2} / 2\right]} & t_{2} \leq t<\infty\end{cases}
$$

Now we investigate the behavior of some systems with a Bathtub failure model, with an exponentially distributed repair time. For the aim of the study, the hazard rate function in term of probability terms can be given as

$$
\begin{aligned}
h(x, x+\delta x) & =\operatorname{Pr}\{x<X \leq x+\delta x \mid X>x\} \\
& =\frac{R(x)-R(x+\delta x)}{R(x)}=1-\frac{R(x+\delta x)}{R(x)}
\end{aligned}
$$


where the continuous distribution can be treated as a discrete distribution. The reliability function involved in (7) is given in (6). We assume that the hazard rate is such that some components survive into the third phase. Otherwise the third phase is irrelevant.

\section{The mean time to failure}

The mean time to failure (MTTF) is an important factor in our study. It is used in evaluating the asymptotic value, i. e. what is called the steady state. This measure, in fact, scales our numerical results. As we have mentioned previously, the mean time to failure of a system, in general, is given by the formula

$M T T F=\int_{0}^{\infty} \mathrm{R}(\mathrm{t}) \mathrm{dt}$.

The reliability function $R(t)$ of the Bathtub failure model is given in (6). From (6) and (7), the mean time to failure MTTF of the system will be given by

$$
M T T F=\left\{\begin{array}{l}
\int_{0}^{t_{1}} e^{-\left(a_{1} t-\frac{1}{2} t^{2}\right)} d t \\
+\int_{t_{1}}^{t_{2}} e^{-\left(a_{1} t_{1}-\frac{1}{2} t^{2}\right)} e^{-a_{2}\left(t-t_{1}\right)} d t \\
+\int_{t_{2}}^{\infty} e^{-\left(a_{1} t_{1}-\frac{1}{2} b t_{1}{ }^{2}\right)} e^{-a_{2}\left(t_{2}-t_{1}\right)} e^{-\left[a_{3}\left(t-t_{2}\right)+b_{3}\left(t-t_{2}\right)^{2} / 2\right]} d t
\end{array}\right.
$$

In (9), in the last integrations, the first two factors are constant. The third factor, however, can be written in a simple way by using a proper transformation. To show this, let us rewrite this factor as

$$
\varphi=\int_{t_{2}}^{\infty} e^{-\left[a_{3}\left(t-t_{2}\right)+b_{3}\left(t-t_{2}\right)^{2} / 2\right]} d t
$$

Then by putting $\mathrm{t}-\mathrm{t} 2 .=\mathrm{u}$ in the integration (9) and rewriting it in a different order, one gets

$$
\varphi=e^{\frac{b_{3}}{2}\left(\frac{a_{3}}{b_{3}}\right)^{2}} \int_{0}^{\infty} e^{-\frac{1}{2}\left[\sqrt{b_{3}}\left(u+\frac{a_{3}}{b_{3}}\right)\right]^{2}} d u
$$

$\sqrt{b_{3}}\left(u+\frac{a_{3}}{b_{3}}\right)=z$ in (11) the term (10) becomes
Once again put

$$
\varphi=\frac{1}{\sqrt{b_{3}}} e^{\frac{a_{3}^{3}}{2 b_{3}}} \int_{\frac{a_{3}}{\sqrt{b_{3}}}}^{\infty} e^{-0.5 z^{2}} d z
$$

The last expression related to normal distribution. If we put (12) into (9), then the MTTF becomes of the form: 


$$
M T T F=\left\{\begin{array}{l}
\int_{0}^{t_{1}} e^{-\left(a_{1} t-\frac{1}{2} t^{2}\right)} d t \\
+\int_{t_{1}}^{t_{2}} e^{-\left(a_{1} t_{1}-\frac{1}{2} t^{2}\right)} e^{-a_{2}\left(t-t_{1}\right)} d t \\
+\int_{t_{2}}^{\infty} e^{-\left(a_{1} t_{1}-\frac{1}{2} b t_{1}{ }^{2}\right)} e^{-a_{2}\left(t_{2}-t_{1}\right)} \frac{1}{\sqrt{b_{3}}} e^{\frac{a_{3}^{3}}{2 b_{3}}} \int_{\frac{a_{3}}{\sqrt{b_{3}}} e^{-0.5 z^{2}}}^{\infty} d z
\end{array}\right.
$$

As a way of example, let calculate the MTTF of the following system

\section{Example 1:}

Suppose we have a system with hazard rate function of a Bathtub type represented in figure2. In this hazard rate we have $\mathrm{t} 1=1.0, \mathrm{t} 2=5.0$ and $\mathrm{t} 3=10$. Also, we have $\mathrm{a} 1=0.005$ $\mathrm{a} 2=0.001 \mathrm{a} 2=\mathrm{a} 3$ and $\mathrm{a} 4=0.3$.

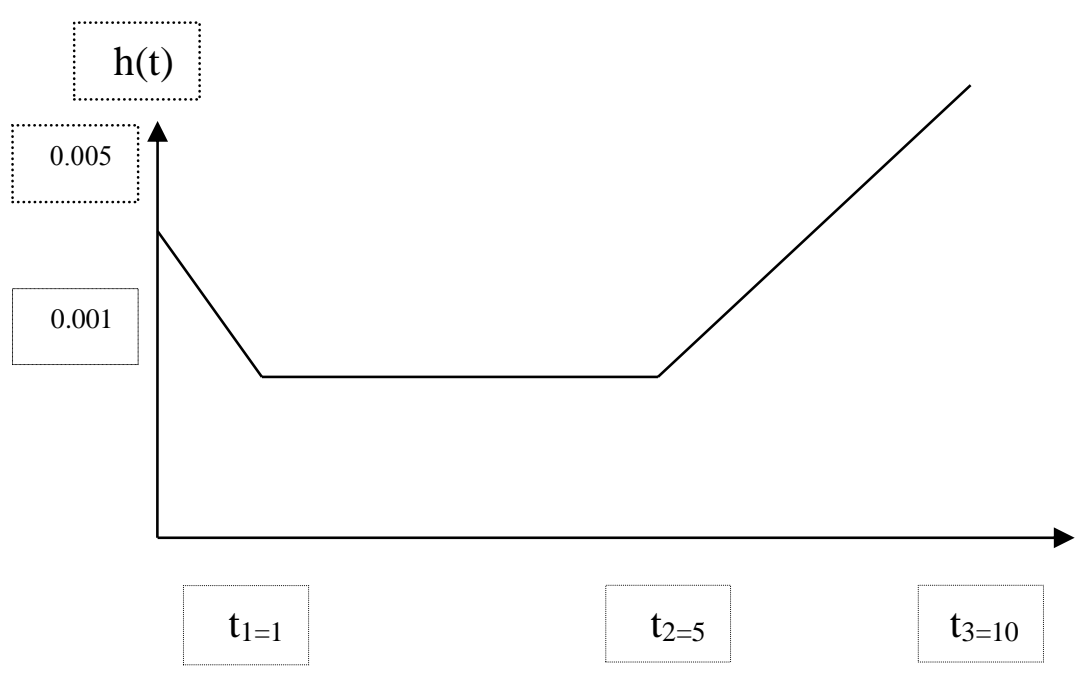

Figure 2 hazard rate function of Example 1

The slopes are computed from (4). The MTTF of the above system, calculated from the expression (13) and the computer is used to carry out this calculation. It is found that the MTTF of the above system is 10.0510898 .

5 Markovian approach to calculate the system availability:

This method, in principle, is used to find out the point availability of the system directly from the hazard rate function. It is found that the hazard function $h(x, x+\delta x)$ can be expressed with reference to the definition of the conditional probability as given in (7). If the limit of (7) exists, then we can write 


$$
Z(x)=\lim _{\delta x \rightarrow 0} \frac{1}{R(x)} \frac{R(x)-R(x+\delta x)}{\delta x}=\frac{f(x)}{R(x)}
$$

The above function $Z(x)$ is called the hazard rate function. If $Z(x)$ exists then (7) rewritten as

$$
\frac{R(x)-R(x+\delta x)}{R(x)}=1-\frac{R(x+\delta x)}{R(x)} \cong Z(x) \delta(x)
$$

The term $Z(x) \delta x$ is approximately the failure probability in the interval $(x, x+\delta x)$. The quantity $Z(x) \delta x$ is used to approximate, where it is appropriate, the failure as well as the repair time distributions. When there are singularities, the original formula (7) is used; otherwise (14) will be used.

The hazard function depends, where it is an up time, on the current age of the component (the item) and on how long it has been down, if it is a down time. So, we only need to take in our consideration the age to see what will happen next. Thus, if we use the current age as a state, the system is Markovian and, therefore, we can describe it by an initial vector and a transition matrix.

It is clear that the hazard function $h(x, x+\delta x)$ can be calculated from $\mathrm{R}(\mathrm{x})$. The term $h(x, x+\delta x)$ measures the mortality near the point $\mathrm{x}$. Meanwhile, the term $1-h(x, x+\delta x)$ is the probability of survival from $\mathrm{x}$ to $(x+\delta x)$. The method is applicable to discrete distributions. But it is also applicable to continuous distributions if they are approximated by discrete ones.

In the continuous case the subdivision between consecutive steps is made very small and is 0.01 in our calculations. So, the continuous distribution can be approximated by a discrete one.

For more details see Ashkar M.Y. [2] pp., 244-245.

\section{The transition matrix:}

To find the availability, a state transition matrix of size $[2(n+1) \times 2(n+1)]$ is needed and it is shown in figure 3. This transition matrix has a special structure. It is distributed into 4 regions to allow transitions among the cases.

The transition matrix is made finite by truncating the life time and the repair time distributions suitably at a very large $n$ to make it easy to deal with. This can be done by making the hazard rate at the state $n$ equal to unity. The same thing is true for the corresponding functions for the down time. For the up time, the hazard function $h(x, x+\delta x)$ will be represented by $\mathrm{p}(\mathrm{x})$. The survival rate will be represented by $1-\mathrm{p}(\mathrm{x})$. The same is for the down time but with $\mathrm{q}(\mathrm{x})$ and $1-q(x)$ instead. For more details see Ashkar M.Y. [2] pp, 244-245.

\section{Initial availability and availability row vector:}

When a new item is put into service at the origin, we start at $t=0$. The availability at this point state is one i.e. $P(0)=1$ And non-availability is zero. Thus, the initial vector is the first essential in calculating the availability at any state time. Given the probability of being up or under repair as illustrated in the transition matrix, we can deduce the point availability, or the vector's availability for the next point. This row vector will replace the initial one for the next step. This can be done by multiplying the vector by the transition matrix. The resultant row vector will show the probability that the system is up or down. Also, it will be used again as the initial availability row vector for the next step. And we repeat the pervious process to get the next point availability and so on. For more details see Ashkar M.Y. [2] pp, 244-245. 
A set of ns+1 element of these refer to up state, denote it by (Ui), The other $n+1$ refer to down state, denote it by (Di). The point availability, simply is $\sum_{i=1}^{2 n} U_{i}$ and the point of non-availability is $\sum_{n+2}^{2 n} D i$ where $\sum_{i=1}^{2 n} U_{i}+\sum_{n+2}^{2 n} D i=1$, for more details see Ashkar, M. Y. [2]

\begin{tabular}{|c|c|c|c|c|c|c|c|c|c|c|c|c|c|c|}
\hline & & & & Up- & Time & & & & & Down & -Time & & & \\
\hline$i=$ & $\left(\begin{array}{ll}n & . .\end{array}\right.$ & .. & 4 & 3 & 2 & 1 & 0 & 0 & 1 & 2 & 3 & $4 \quad .$. & & n) \\
\hline$n$ & 0 & .. & 0 & 0 & 0 & 0 & 0 & 1 & 0 & 0 & 0 & 0 & $\ldots$ & 0 \\
\hline$n-1$ & $1-p_{n-1, n}$ & . & 0 & 0 & 0 & 0 & 0 & $p_{n-1, n}$ & 0 & 0 & 0 & 0 & $\ldots$ & 0 \\
\hline & $\ldots$ & .. &.. &.. & & & & &.. & & & & $\ldots$ & \\
\hline 3 & 0 & .. & $1-p_{34}$ & 0 & 0 & 0 & 0 & $p_{34}$ & 0 & 0 & 0 & 0 & $\ldots$ & 0 \\
\hline 2 & 0 & .. & 0 & $1-p_{23}$ & 0 & 0 & 0 & $p_{23}$ & 0 & 0 & 0 & 0 & $\ldots$ & 0 \\
\hline 1 & 0 & .. & 0 & 0 & $1-p_{12}$ & 0 & 0 & $p_{12}$ & 0 & 0 & 0 & 0 & $\ldots$ & 0 \\
\hline 0 & 0 & .. & 0 & 0 & 0 & $1-p_{01}$ & 0 & $p_{01}$ & 0 & 0 & 0 & 0 & $\ldots$ & 0 \\
\hline 0 & 0 & .. & 0 & 0 & 0 & 0 & $q_{01}$ & 0 & $1-q_{01}$ & 0 & 0 & 0 & $\ldots$ & 0 \\
\hline 1 & 0 & .. & 0 & 0 & 0 & 0 & $q_{12}$ & 0 & 0 & $1-q_{12}$ & 0 & 0 & $\ldots$ & 0 \\
\hline 2 & 0 & .. & 0 & 0 & 0 & 0 & $q_{23}$ & 0 & 0 & 0 & $1-q_{23}$ & & & 0 \\
\hline & 0 & .. & 0 & 0 & 0 & 0 & $q_{34}$ & 0 & 0 & 0 & 0 & $1-q_{34}$ & & 0 \\
\hline$n-1$ & 0 & .. & 0 & 0 & 0 & 0 & $q_{n-1, n}$ & 0 & 0 & 0 & 0 & 0 & & $1-q_{n-1, n}$ \\
\hline$n$ & 0 & .. & 0 & 0 & 0 & 0 & 1 & 0 & 0 & 0 & 0 & 0 & & 0 \\
\hline
\end{tabular}

Figure3 illustrates the nature of the Transition Matrix of size $2(n+1) \times 2(n+1)$ Among states 
Up-Time

Down-Time

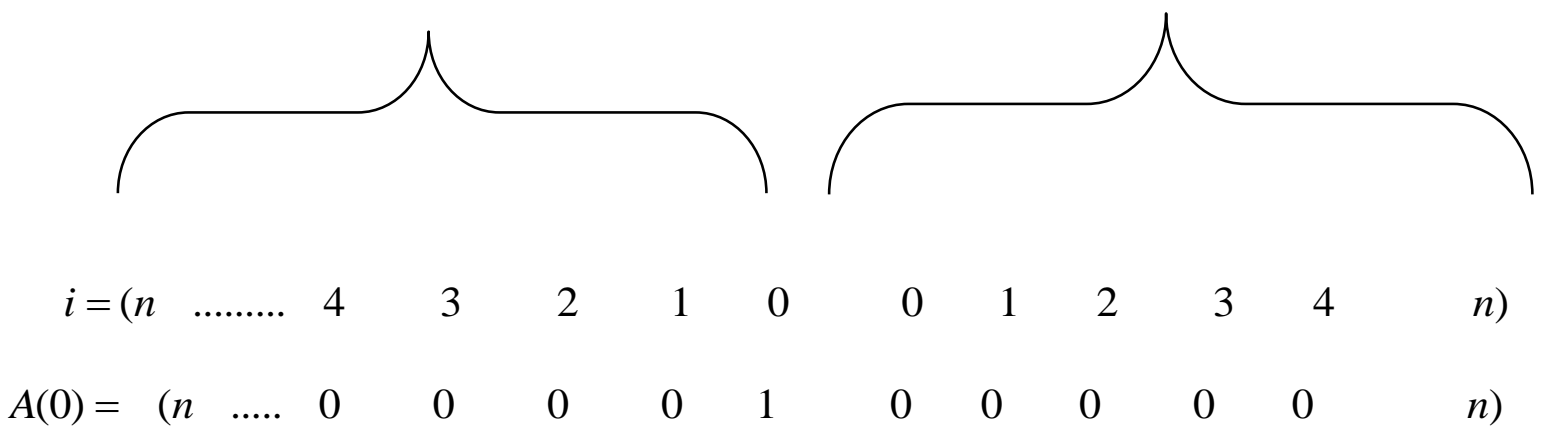

Figure 4 illustrates the $2(n+1)$ initial availability row vector

8 The Bathtub failure and exponential distribution repair

\section{System I:}

In this section the point availability of a system with a Bathtub hazard rate failure, where the repair time is exponentially distributed, which is a special case of Weibull and or Gamma distributions. More specifically, let us suppose that the system operates with the Bath-Tub hazard rate failure as shown in figure 5 . This hazard rate is specified by the following terms:

$$
\begin{array}{ll}
a_{1}=0.5 & t_{1}=2.0 \\
a_{2}=a_{3}=0.1 & t_{2}=5.0 \\
a_{4}=0.2 & t_{3}=10.0
\end{array}
$$

The slopes in this model are calculated from (4). The reliability function of the system is computed directly from (6). The curve of this reliability function is shown in figure 6 From the reliability function, the hazard function can be obtained. Note that the continuous function is approximated by a discrete one, where the subdivision between the consecutive steps is 0.01 This, however, does not rule out the possibility of using the continuous formula of the hazard rate function i.e. the LHS of the expression (7) for the discrete case and the RHS of the (7) for the continuous case. The mean of the repair time is assumed to be 0.25 ; i.e. an exponential with $\lambda=4$. The above Markovian method is applied to obtain the point availability. The result converted to curve and it is shown in figure7. The mean time to failure of this system is computed in a similar way to example.1. The mean time to failure in this case is 5.0489912 . The asymptotic result gives

$\operatorname{Lim} P A(t)=\frac{5.0489912}{5.0489912+0.25}=0.9528212$ 


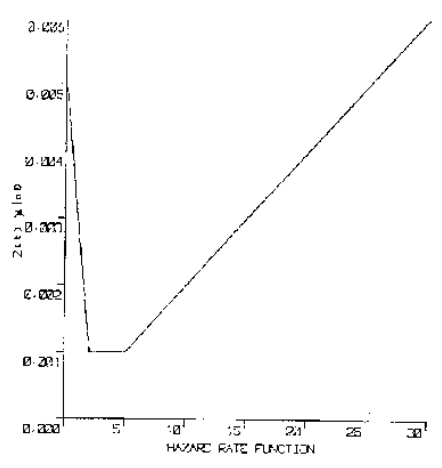

Figure 5: Hazard Rate Function of System I

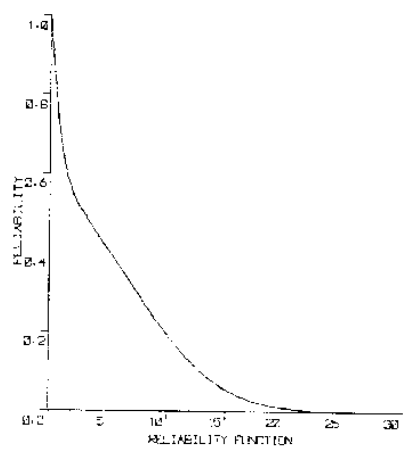

Figure 6: Reliability Function of System I

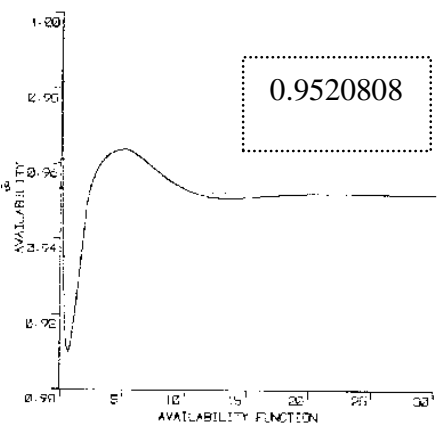

Figure 7: Availability Function of System I

This Asymptotic result is 5.0489912 and the point availability of the system computed by Markoian method is PA (30) $=0.9520808$. This has absolute error between the two results of 0.0006212 , which is $0.06212 \%$

\section{System II:}

By following the same steps achieved in system I, system II will be considered. The bathtub hazard rate function, which is illustrated in figure 8 , is specified as follows:

$$
\begin{array}{ll}
a_{1}=0.5 & t_{1}=0.1 \\
a_{2}=a_{3}=0.1 & t_{2}=5.0 \\
a_{4}=0.2 & t_{3}=10.0
\end{array}
$$

The repair time is exponentially distributed with mean 0.25 . The curve of reliability function is shown in figure 9.

The above Markovian method is applied to obtain the point availability. The result converted to curve and it is shown in figure10. The mean time to failure of this system is computed in a similar way to example.1. The mean in this case is 7.1013871 . The asymptotic result gives

$$
\operatorname{Lim}_{t \rightarrow \infty} P A(t)=\frac{7.1013871}{7.1013871+0.25}=0.9659928
$$

This Asymptotic result is 0.9659928 and the point availability of the system computed by Markoian method is PA (30) $=0.9595632$. This has absolute error between the two results of 0.0064296 , which is $0.643 \%$ 


\section{System III:}

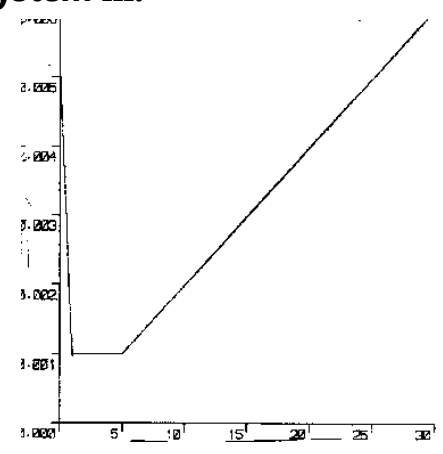

Figure 8: Hazard rate

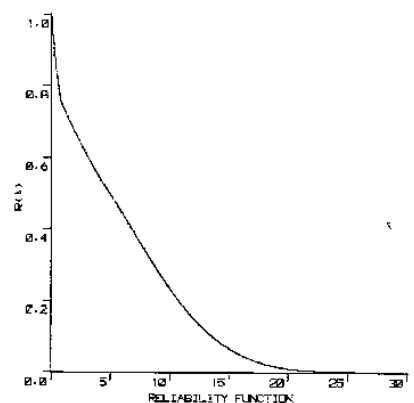

Figure9: Reliability

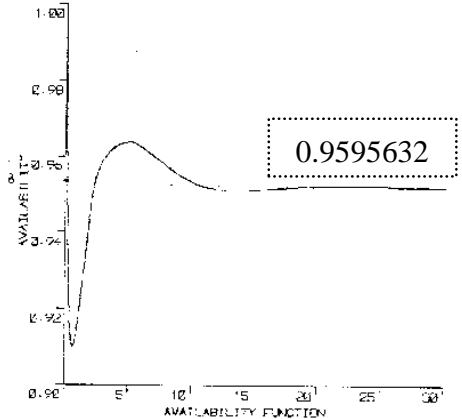

Figure 10: Availability

Function of System II

Function of System II

Function of System II

As third and final system, system III with hazard rate function is specified below is considered. The same above steps of system I and system II are achieved. The bathtub hazard rate function, which is illustrated in figure 11 , is given as follows:

$$
\begin{array}{ll}
a_{1}=0.005 & t_{1}=0.1 \\
a_{2}=a_{3}=0.001 & t_{2}=5.0 \\
a_{4}=0.300 & t_{3}=10.0
\end{array}
$$

The repair time is exponentially distributed with mean 0.25 . The curve of reliability function is shown in figure 12.

The above Markovian method is applied to obtain the point availability. The result converted to curve and it is shown in figure13. The mean time to failure of this system is computed in a similar way to example.1. The mean in this case is 10.0510898 . The asymptotic result gives

$$
\operatorname{Lim} P A(t)=\frac{10.0510898}{10.0510898+0.25}=0.9757307
$$

This Asymptotic result is 0.9757307 and the point availability of the system computed by Markoian method is PA (25) $=0.9765889$. This has absolute error between the two results of 0.0008582 , which is $0.0858 \%$

\begin{tabular}{|l|l|l|}
\hline & \\
\hline Figure 11: Hazard rate & Figure12: Reliability & \\
\hline Function of System III & Function of System III & Function of System III \\
\hline
\end{tabular}


A comparison between the above systems, give the following contrast

\begin{tabular}{|c|c|c|c|}
\hline \multicolumn{2}{|c|}{$\begin{array}{l}\text { Specification of Bathtub } \\
\text { Hazard rate failure }\end{array}$} & $\begin{array}{l}\text { Mean } \\
\text { Time to } \\
\text { failure }\end{array}$ & Asymptotic results \\
\hline $\begin{array}{l}a_{1}=0.5 \\
a_{2}=a_{3}=0.1 \\
a_{4}=0.2\end{array}$ & $\begin{array}{l}t_{1}=2.0 \\
t_{2}=5.0 \\
t_{3}=10.0\end{array}$ & 5.0489912 & $\operatorname{Lim}_{t \rightarrow \infty} P(t)=\frac{5.0489912}{5.0489912+0.25}=0.9528212$ \\
\hline $\begin{array}{l}a_{1}=0.5 \\
a_{2}=a_{3}=0.1 \\
a_{4}=0.2\end{array}$ & $\begin{array}{l}t_{1}=0.1 \\
t_{2}=5.0 \\
t_{3}=10.0\end{array}$ & 7.1013871 & $\operatorname{Lim}_{t \rightarrow \infty} P A(t)=\frac{7.1013871}{7.1013871+0.25}=0.9659928$ \\
\hline $\begin{array}{l}a_{1}=0.005 \\
a_{2}=a_{3}=0.001 \\
a_{4}=0.300\end{array}$ & $\begin{array}{l}t_{1}=0.1 \\
t_{2}=5.0 \\
t_{3}=10.0\end{array}$ & $\begin{array}{l}10.051089 \\
8\end{array}$ & $\operatorname{Lim} P A(t)=\frac{10.0510898}{10.0510898+0.25}=0.9757307$ \\
\hline
\end{tabular}

\section{To sum up:}

It is clear that the last system, system III is the best since it reaches the asymptotic state faster than the other two and has bigger mean time to failure which means it takes longer time to fail. This mean its reliability is better than other two. From the above study of systems with different bathtub, it can be concluded that the point availability of the system depends on the nature of its Bathtub hazard rate function. It settles down to the steady-state within a short time. The repair time distribution which is an exponential, speed up the process to equilibrium, but the nature of the "Bathtub" curve can give practically no oscillation, or a short large one

\section{References}

1. Ascher, H. and Feingold, H. "The Repairable System Reliability", Marcel Dekker, New York, 1984.

2. Ashkar, M. Y. " Markovian Method of Finding Point of Availability of Repairable Systems", Journal of Emerging Trends in Computing and information Science, CIS Journal, pp. 243250, Volume 2 No.5 May 2011.

3. Ashkar, M. Y. "A Stochastic Approach to Problems of the Königsberg Bridges", CIS Journal of Emerging Trends in Computing and Information Sciences, Vol. 3 No.2 February 2012.

4. Baker, C. "Numerical Treatment of Integral Equations", Oxford University Press, London 1977.

5. Barlow, R. and L. Hunter, "Optimum Preventive Maintenance Policies", Operations Research, Vol. 8, pp. 90-100, 1960.

6. Barlow, R. and L. Hunter, "Reliability Analysis of a One-unit System", Operations Research, Vol. 9, pp. 200-2008, 1961. 
7. Barlow, R, and Proschan, F. "Statistical theory of Reliability Theory of Reliability and Life Testing", John Wiley, New York, 1975.

8. Cox, D. R. "Renewal Theory", Methuen, London, 1962.

9. Cha, J. H. "An extended model for optimal burn-in procedures," IEEE Transactions on Reliability, vol. 55, no. 2, pp. 189-198, 2006

10. Davis, D.J., "An Analysis of Some Failure Data", J. Am. Stat. Assoc., Vol. 47, 1952.

11. Elliott, D. and Warent, W.G. "An Algorithm for the Numerical Solution of Linear Integral Equation", Int. Cent. Bull., Vol. 6, pp.207-224, 1967.

12. Elsayed, E., "Reliability Engineering", Addison Wesley, Reading, MA, 1996.

13. Fox. L., and Goodwin, E. T., "The Numerical Solution of Non-Singular Integral Equation", Phil. Trans. Roy. Soc. A245, PP 501-534, 1953.

14. Kapur, K.C. and L.R. Lamberson, "Reliability in Engineering Design", John Wiley \& Sons, Inc., New York, 1977.

15. Kececioglu, D., "Reliability Engineering Handbook", Volume 1, Prentice Hall, Inc., New Jersey, 1991.

16. Kececioglu, D., "Maintainability, Availability, \& Operational Readiness Engineering", Volume 1, Prentice Hall PTR, New Jersey, 1995.

17. Kijima, M. and Sumita, N., "A Useful Generalization of Renewal Theory: counting process governed by nonnegative Markovian increments", Journal of Applied Probability, 23, 71-88, 1986.

18. Kijima, M., "Some results for repairable systems with general repair", Journal of Applied Probability, 20, pp.851-859, 1989.

19. Knuth, D.E., "The Art of Computer Programming", Volume 2, Semi numerical Algorithms, Third Edition, Addison-Wesley, 1998.

20. L'Ecuyer, P., Communications of the ACM, Vol. 31, pp.724-774, 1988.

21. L'Ecuyer, P., Proceedings of the 2001 Winter Simulation Conference, pp.95-105, 2001.

22. Leemis, L.M., "Reliability - Probabilistic Models and Statistical Methods", Prentice Hall, Inc., Englewood Cliffs, New Jersey, 1995.

23. Lewis, E., "Introduction to Reliability Engineering", John Wiley, New York,1987

24. Mettas, A., "Reliability Allocation and Optimization for Complex Systems", Proceedings of the Annual Reliability \& Maintainability Symposium, 2000.

25. Shooman M.L. Probabilistic Reliability; An Engineering Approaches, McGraw Hill, New York 1968

26. Tillman, F.A., "Numerical Evaluation of Instantaneous Availability", IEEE, Transactions on Reliability, Vol. R32, No.1, 1983.

27. Xie, M, Tang $Y$, and T. N. Goh T. N., "A modified Weibull extension with bathtub-shaped failure rate function," Reliability Engineering and System Safety, vol. 76, no. 3, pp. 279-285, 2002. 